\title{
ENTROPY FUNCTIONALS AND THEIR EXTREMAL VALUES FOR SOLVING THE STIELTJES MATRIX MOMENT PROBLEM
}

\author{
YU. M. DYUKAREV
}

Dedicated to Yurii Arlinskii on occasion of his 70th birthday

\begin{abstract}
Entropy functionals and their extremal values for solving the Stieltjes matrix moment problem are defined and investigated for the first time. Explicit formulas for the extremal values of the entropy over the set of solutions of the Stieltjes matrix moment problem are obtained. A geometric interpretation in terms of Weyl matrix intervals is presented.
\end{abstract}

\section{INTRODUCTION}

Entropy functionals and their extremal values have been studied by many authors (see, for example, [1], [2], [3], [5]). But similar functionals were not considered for solution interpolation problems in the matrix Stieltjes class. In this paper, entropy functionals over solutions of the Stieltjes matrix moment problem are defined and studied for the first time.

Given integers $m, n \geq 1$, we let $\mathbb{C}^{m}$ denote the linear space of columns of complex numbers $x=\operatorname{col}\left(x_{1} x_{2} \ldots x_{m}\right)$ of size $m$ equipped with the inner product $(x, y)=$ $\sum_{j=1}^{m} \bar{x}_{i} y_{j}$. Let $\mathbb{C}^{m \times n}$ be the set of complex matrices with $m$ rows and $n$ columns. Denote by $\mathbb{C}_{H}^{m \times m}$ the set of all Hermitian matrices. A Hermitian matrix $A$ is called nonnegative if $(x, A x) \geq 0 \forall x \in \mathbb{C}^{m}$. By $\mathbb{C}_{>}^{m \times m}$ denote the set of nonnegative matrices. A nonnegative matrix $A$ is called positive if $(x, A x)>0$ for any nonzero vector $x \in \mathbb{C}^{m}$. Let $\mathbb{C}_{>}^{m \times m}$ be the set of positive matrices. By $I_{m} \in \mathbb{C}^{m \times m}$ denote the identity matrix and by $O_{m \times n} \in \mathbb{C}^{m \times n}$ denote the zero matrix. We will often omit the subscripts of the identity matrix and the zero matrix if these subscripts are clear from the context. For Hermitian matrices $A, B$ we write $A>B(A \geq B)$ if $A-B \in \mathbb{C}_{>}^{m \times m}\left(A-B \in \mathbb{C}_{\geq}^{m \times m}\right)$. If the matrix $A$ is invertible then by $A^{-*}$ denote the matrix $\left(A^{-1}\right)^{*}$. If $f(z)$ is a matrix function (MF) then by $f^{*}(z)$ denote the MF $(f(z))^{*}$. Let $f(z)$ be an invertible MF. By $f^{-1}(z)$ and $f^{-*}(z)$ denote MFs $(f(z))^{-1}$ and $\left((f(z))^{-1}\right)^{*}$ respectively. By definition $z=|z| \exp (i \arg z),-\pi<\arg z \leq \pi$ and $\sqrt{z}=\sqrt{|z|} \exp \left(\frac{i \arg z}{2}\right)$.

We will also write $\mathbb{C}_{+}=\{z \in \mathbb{C}: \operatorname{Im} z>0\}, \mathbb{C}_{-}=\{z \in \mathbb{C}: \operatorname{Im} z<0\}, \mathbb{R}_{+}=\{x \in \mathbb{R}:$ $x \geq 0\}$ and $\mathbb{R}_{-}=\{x \in \mathbb{R}: x<0\}$.

Denote by $\mathfrak{B}$ the $\sigma$-algebra of Borel subsets of the real line $\mathbb{R}$. A mapping $\sigma: \mathfrak{B} \rightarrow$ $\mathbb{C}_{\geq}^{m \times m}$ is called a nonnegative matrix measure if

$$
\sigma\left(\bigcup_{j=1}^{\infty} A_{j}\right)=\sum_{j=1}^{\infty} \sigma\left(A_{j}\right)
$$

for any infinite sequence $\left(A_{j}\right)_{j=1}^{\infty}$ of pairwise disjoint Borel subsets of $\mathbb{R}$.

2020 Mathematics Subject Classification. 30E05, 44A60, 47A57.

Key words and phrases. Stieltjes moment problem, entropy functionals, maximum entropy. 
We first recall some facts about the Stieltjes matrix moment problem (see [6]-[10]). Let $\left(s_{j}\right)_{j=0}^{2 n+1}$ be an arbitrary sequence of complex $m \times m$ matrices. We consider the following block matrices:

$$
\begin{aligned}
& H_{1}=\left(s_{j+k}\right)_{j, k=0}^{n}, \quad H_{2}=\left(s_{j+k+1}\right)_{j, k=0}^{n}, \quad T_{1}=\left(\begin{array}{cc}
O_{m n \times m} & I_{m n} \\
O_{m \times m} & O_{m \times m n}
\end{array}\right), \\
& R_{1}(z)=\left(I-z T_{1}\right)^{-1}, \quad u_{2}=\operatorname{col}\left(-s_{0}-s_{1} \ldots-s_{n}\right), \quad u_{1}=T_{1}^{\prime} u_{2}, \quad v_{1}=\left(\begin{array}{c}
I_{m} \\
O_{m n \times m}
\end{array}\right) .
\end{aligned}
$$

Assume that the block matrices $H_{1}$ and $H_{2}$ satisfy the following conditions:

$$
H_{1}>O, \quad H_{2}>O \text {. }
$$

In the Stieltjes matrix moment problem it is required to describe all matrix-valued nonnegative measures $\sigma$ on the half-axis $\mathbb{R}_{+}$such that

$$
s_{j}=\int_{\mathbb{R}_{+}} t^{j} \sigma(d t), \quad 0 \leq j \leq 2 n, \quad s_{2 n+1} \geq \int_{\mathbb{R}_{+}} t^{2 n+1} \sigma(d t) .
$$

Let $\mathcal{M}_{+}$denote the set of all solutions $\sigma$ to the Stieltjes matrix moment problem. Under the above assumptions it is known that $\mathcal{M}_{+} \neq \varnothing$. With each solution of the Stieltjes matrix moment problem we associate a MF as follows:

$$
s(z)=\int_{\mathbb{R}_{+}} \frac{\sigma(d t)}{t-z}, \quad \sigma \in \mathcal{M}_{+} .
$$

By $\mathcal{F}_{+}$denote the set of associated MFs. It is obvious that associated MFs are holomorphic MFs in $\mathbb{C} \backslash \mathbb{R}_{+}$. The Stieltjes inversion formula establishes a one-to-one correspondence between $\mathcal{F}_{+}$and $\mathcal{M}_{+}$.

Let

$$
J=\left(\begin{array}{cc}
O_{m \times m} & -i I_{m \times m} \\
i I_{m \times m} & O_{m \times m}
\end{array}\right), \quad J_{\pi}=\left(\begin{array}{cc}
O_{m \times m} & I_{m \times m} \\
I_{m \times m} & O_{m \times m}
\end{array}\right) .
$$

The pair of meromorphic $m \times m \operatorname{MF} \operatorname{col}(p(z) q(z))$ in $\mathbb{C} \backslash \mathbb{R}_{+}$is said to be Stieltjes if for this pair there exists a discrete the set of points $\mathcal{D}_{p q}$ in $\mathbb{C} \backslash \mathbb{R}_{+}$such that

(1) $p^{*}(z) p(z)+q^{*}(z) q(z)>O, z \in \mathbb{C} \backslash\left\{\mathbb{R}_{+} \cup \mathcal{D}_{p q}\right\}$.

(2) $\left(p^{*}(z), q^{*}(z)\right) \frac{J}{i(\bar{z}-z)}\left(\begin{array}{c}p(z) \\ q(z)\end{array}\right) \geq O, z \in \mathbb{C} \backslash\left\{\mathbb{R} \cup \mathcal{D}_{p q}\right\}$.

(3) $\left(p^{*}(z), q^{*}(z)\right) J_{\pi}\left(\begin{array}{c}p(z) \\ q(z)\end{array}\right) \geq O, z \in\{z \in \mathbb{C}: \Re z<0\} \backslash \mathcal{D}_{p q}$.

On the set of Stieltjes pairs, we introduce the equivalence ratio: the pairs $\operatorname{col}\left(p_{1}(z) q_{1}(z)\right)$ and $\operatorname{col}\left(p_{2}(z) q_{2}(z)\right)$ are said to be equivalent if there exists a MF $Q(z)$ such that the MF $Q(z),(Q(z))^{-1}$ are both meromorphic in $\mathbb{C} \backslash \mathbb{R}_{+}$and

$$
p_{1}(z)=p_{2}(z) Q(z), \quad q_{1}(z)=q_{2}(z) Q(z) .
$$

The set of equivalence classes of Stieltjes pairs will be denoted by $\mathcal{S}_{\infty}$.

A polynomial MF

$$
U_{1}(z)=\left(\begin{array}{cc}
\alpha_{1}(z) & \beta_{1}(z) \\
\gamma_{1}(z) & \delta_{1}(z)
\end{array}\right)=\left(\begin{array}{cc}
I+z v_{1}^{*} R_{1}(z) H_{2}^{-1} u_{2} & -z v_{1}^{*} R_{1}(z) H_{1}^{-1} v_{1} \\
u_{2}^{*} R_{1}(z) H_{2}^{-1} u_{2} & I-z u_{1}^{*} R_{1}(z) H_{1}^{-1} v_{1}
\end{array}\right)
$$

is called the resolvent matrix the Stieltjes moment problem.

The formula

$$
s(z)=\left(\gamma_{1}(z) p(z)+\delta_{1}(z) q(z)\right)\left(\alpha_{1}(z) p(z)+\beta_{1}(z) q(z)\right)^{-1}
$$

establishes a bijective correspondence between $\mathcal{F}_{+}$and $\mathcal{S}_{\infty}$.

Substituting the Stieltjes pairs col $(I O)$ and $\operatorname{col}(O I)$ in (1), we obtain extremal MFs

$$
s_{F}(z)=\gamma_{1}(z) \alpha_{1}^{-1}(z) \in \mathcal{F}_{+}, \quad s_{K}(z)=\delta_{1}(z) \beta_{1}^{-1}(z) \in \mathcal{F}_{+} .
$$


Suppose that $t_{0} \in \mathbb{R}_{-}$. The matrix interval $\left[s_{F}\left(t_{0}\right), s_{K}\left(t_{0}\right)\right]$ is called the matrix Weyl interval (see [6]-[10]). We can prove that $\left\{s\left(x_{0}\right): s \in \mathcal{F}_{+}\right\}=\left[s_{F}\left(t_{0}\right), s_{K}\left(t_{0}\right)\right]$.

From (1) it follows that $\left(s(z)-s^{*}(z)\right) / i \geq O, z \in \mathbb{C}_{+}$. Consequently, at almost all points $t \geq 0$ exist nontangential limits $s(t)=\lim _{y \rightarrow+0} s(t+i y)$. Let $t_{0}$ be a point belongs to $\mathbb{R}_{-}$. For any $s$ belongs to $\mathcal{F}_{+}$the entropy functional $\mathcal{I}\left(s ; t_{0}\right)$ is defined by the formula

$$
\mathcal{I}\left(s ; t_{0}\right)=\int_{0}^{+\infty} \ln \left(\frac{s(t)-s^{*}(t)}{2 i}\right) \frac{\sqrt{-t_{0}}}{\pi \sqrt{t}\left(t-t_{0}\right)} d t .
$$

The main result in our paper is as follows.

Theorem 1. The entropy functional has an upper bound

$$
\mathcal{I}\left(s ; t_{0}\right) \leq \ln \operatorname{det} \frac{s_{K}\left(t_{0}\right)-s_{F}\left(t_{0}\right)}{4} \quad \forall s \in \mathcal{F}_{+},
$$

with equality if and only if

$$
\begin{gathered}
\tilde{s}(z)=\left(\gamma_{1}(z) \tilde{p}(z)+\delta_{1}(z) \tilde{q}(z)\right)\left(\alpha_{1}(z) \tilde{p}(z)+\beta_{1}(z) \tilde{q}(z)\right)^{-1} \in \mathcal{F}_{+}, \\
\left(\begin{array}{c}
\tilde{p}(z) \\
\tilde{q}(z)
\end{array}\right)=\left(\begin{array}{c}
\beta_{1}^{*}\left(t_{0}\right) / \sqrt{t_{0}} \\
\alpha_{1}^{*}\left(t_{0}\right) / \sqrt{z}
\end{array}\right) Q(z) \in \mathcal{S}_{\infty} .
\end{gathered}
$$

Moreover, the matrix $\tilde{s}\left(t_{0}\right)$ coincides with the center of the matrix Weyl interval $\left[s_{F}\left(t_{0}\right), s_{K}\left(t_{0}\right)\right]$, i.e.,

$$
\tilde{s}\left(t_{0}\right)=\frac{s_{K}\left(t_{0}\right)+s_{F}\left(t_{0}\right)}{2} .
$$

\section{Entropy functionals for the Hamburger matrix moment Problem}

We first recall some facts about the Hamburger matrix moment problem (see, for example, [4], [5], [12], [13]). Let $\left(w_{j}\right)_{j=0}^{2 n}$ be an arbitrary sequence of complex $m \times m$ matrices. We consider the following block matrices

$$
\begin{aligned}
& H=\left(w_{j+k}\right)_{j, k=0}^{n}, \quad T=\left(\begin{array}{cc}
O_{m n \times m} & I_{m n} \\
O_{m \times m} & O_{m \times m n}
\end{array}\right), \quad R(w)=(I-w T)^{-1}, \\
& u=\operatorname{col}\left(O-w_{0}-w_{1} \ldots-w_{n-1}\right), \quad v=\left(\begin{array}{c}
I_{m} \\
O_{m n \times m}
\end{array}\right) .
\end{aligned}
$$

Assume that the block matrix $H$ is positive. In the Hamburger matrix moment problem it is required to describe all the matrix-valued nonnegative measures $\tau$ such that

$$
w_{j}=\int_{\mathbb{R}} t^{j} \tau(d t), \quad 0 \leq j \leq 2 n-1, \quad w_{2 n} \geq \int_{\mathbb{R}} t^{2 n} \tau(d t) .
$$

Let $\mathcal{M}$ denote the set of solutions to problem (3). Under the above assumptions, $\mathcal{M} \neq \varnothing$. With each matrix measure $\tau \in \mathcal{M}$ we associate a MF as follows:

$$
f(w)=\int_{\mathbb{R}} \frac{\tau(d t)}{t-w} .
$$

By $\mathcal{F}$ denote the set of associated MFs $f$. It is obvious that associated MFs are holomorphic MFs in $\mathbb{C}_{+}$. The Stieltjes inversion formula establishes a one-to-one correspondence between $\mathcal{F}$ and $\mathcal{M}$.

A pair of meromorphic $m \times m \operatorname{MF} \operatorname{col}(p(w) q(w))$ in $\mathbb{C}_{+}$is said to be Nevanlinna if for this pair there exists the discrete set of points $\mathcal{D}_{p q}$ in $\mathbb{C}_{+}$such that

(1) $p^{*}(w) p(w)+q^{*}(w) q(w)>O, w \in \mathbb{C}_{+} \backslash \mathcal{D}_{p q}$.

(2) $\left(p^{*}(w) q^{*}(w)\right) \frac{J}{i(\bar{z}-z)}\left(\begin{array}{c}p(w) \\ q(w)\end{array}\right) \geq O, w \in \mathbb{C}_{+} \backslash \mathcal{D}_{p q}$. 
On the set of Nevanlinna pairs, we introduce the equivalence ratio: the pairs

$$
\operatorname{col}\left(p_{1}(w) q_{1}(w)\right) \text { and } \operatorname{col}\left(p_{2}(w) q_{2}(w)\right)
$$

are said to be equivalent if there exists a $\operatorname{MF} Q(w)$ such that the $\operatorname{MF} Q(w),(Q(w))^{-1}$ are both meromorphic in $\mathbb{C}_{+}$and

$$
p_{1}(w)=p_{2}(w) Q(w), \quad q_{1}(w)=q_{2}(w) Q(w) .
$$

The set of equivalence classes of Nevanlinna pairs will be denoted by $\mathcal{R}_{\infty}$.

A polynomial $\mathrm{MF}$

$$
U(w)=\left(\begin{array}{cc}
\alpha(w) & \beta(w) \\
\gamma(w) & \delta(w)
\end{array}\right)=\left(\begin{array}{cc}
I+z v^{*} R(w) H^{-1} u & -z v^{*} R(w) H^{-1} v \\
z u^{*} R(w) H^{-1} u & I-z u^{*} R(w) H^{-1} v
\end{array}\right)
$$

is called the resolvent matrix the Hamburger moment problem.

The formula

$$
f(w)=(\gamma(w) p(w)+\delta(w) q(w))(\alpha(w) p(w)+\beta(w) q(w))^{-1}
$$

establishes a bijective correspondence between $\mathcal{F}$ and $\mathcal{R}_{\infty}$.

By $\mathbf{S}$ denote the set of $m \times m$ MFs $S(w)$ which are analytic and contractive (i.e., $\left.S^{*}(w) S(w) \leq I_{m}\right)$ in $\mathbb{C}_{+}$.

It is well known that the formula

$$
p(w)=(I+S(w)) Q(w), \quad q(w)=i(I-S(w)) Q(w)
$$

establishes a bijective correspondence between $\mathbf{S}$ and $\mathcal{R}_{\infty}$. Here MFs $Q(z),(Q(z))^{-1}$ are both meromorphic in $\mathbb{C}_{+}$.

By definition, put

$$
\begin{aligned}
E(w) & =\alpha(w)+i \beta(w), & & F(w)=\alpha(w)-i \beta(w), \\
G(w) & =\gamma(w)+i \delta(w), & & H(w)=\gamma(w)-i \delta(w) .
\end{aligned}
$$

Combining (6), (7), (8), and (9), we get

$$
f(w)=(G(w)+H(w) S(w))(E(w)+F(w) S(w))^{-1} .
$$

From (4) follows that $\left(f(w)-f^{*}(w)\right) / i \geq O, w \in \mathbb{C}_{+}$. Consequently, at almost all points $x \in \mathbb{R}$ exist nontangential limits $f(x)=\lim _{y \rightarrow+0} f(x+i y)$. Let $w_{0}=x_{0}+i y_{0}$ be a point belongs to $\mathbb{C}_{+}$. For any $f$ belongs to $\mathcal{F}$ the entropy functional $\mathcal{I}\left(f ; w_{0}\right)$ is defined by the formula

$$
\mathcal{I}\left(f ; w_{0}\right)=\int_{\mathbb{R}} \ln \left(\operatorname{det} \frac{f(x)-f^{*}(x)}{2 i}\right) \frac{y_{0}}{\pi\left(\left(x-x_{0}\right)^{2}+y_{0}^{2}\right)} d x .
$$

The following theorem was proved in [5].

Theorem 2. The entropy functional has an upper bound

$$
\mathcal{I}\left(f ; w_{0}\right) \leq \ln \operatorname{det}\left(2 i\left(\bar{w}_{0}-w_{0}\right) v^{*} R\left(w_{0}\right) H^{-1} R^{*}\left(w_{0}\right) v\right)^{-1} \quad \forall f \in \mathcal{F},
$$

with equality if and only if

$$
\begin{gathered}
\tilde{f}(w)=(G(w)+H(w) \tilde{S})(E(w)+F(w) \tilde{S})^{-1} \in \mathcal{F}, \\
\tilde{S}=-F^{*}\left(w_{0}\right) E^{-*}\left(w_{0}\right) \in \mathbf{S} .
\end{gathered}
$$

In other words,

$$
\begin{gathered}
\tilde{f}(w)=(\gamma(w) \tilde{p}(w)+\delta(w) \tilde{q}(w))(\alpha(w) \tilde{p}(w)+\beta(w) \tilde{q}(w))^{-1} \in \mathcal{F} \\
\left(\begin{array}{c}
\tilde{p}(w) \\
\tilde{q}(w)
\end{array}\right)=\left(\begin{array}{c}
-\beta^{*}\left(w_{0}\right) \\
\alpha^{*}\left(w_{0}\right)
\end{array}\right) Q(w) \in \mathcal{R}_{\infty} .
\end{gathered}
$$




\section{Entropy functionals For the Symmetric Hamburger moment PRoblem}

Let $\left(w_{j}\right)_{j=0}^{4 n+2}$ be a sequence of complex $m \times m$ matrices such that

$$
H=\left(w_{j+k}\right)_{j, k=0}^{2 n+1}>O .
$$

Corresponding moment problem (3) is said to be the symmetric Hamburger matrix moment problem if $w_{2 j+1}=O_{m \times m}, \quad j=0, \ldots, 2 n$. The symmetric moment problem we will study in these chapter.

If we replace $w_{2 j}, j=0, \ldots, 2 n+1$ by $s_{j}$, we obtain a sequence

$$
s_{0}, O, s_{1}, O, s_{2}, O, \ldots, O, s_{2 n+1} .
$$

Given the sequence (12) of $m \times m$ matrices, we construct the following block matrices:

$$
\begin{aligned}
& H=\left(\begin{array}{ccccccc}
s_{0} & O & s_{1} & O & \ldots & s_{n} & O \\
O & s_{1} & O & s_{2} & \ldots & O & s_{n+1} \\
s_{1} & O & s_{2} & O & \ldots & s_{n+1} & O \\
O & s_{2} & O & s_{3} & \ldots & O & s_{n+2} \\
\vdots & \vdots & \vdots & \vdots & \ddots & \vdots & \vdots \\
s_{n} & O & s_{n+1} & O & \ldots & s_{2 n} & O \\
O & s_{n+1} & O & s_{n+2} & \cdots & O & s_{2 n+1}
\end{array}\right) \in \mathbb{C}^{(2 n+2) m \times(2 n+2) m}, \\
& T=\left(\begin{array}{cc}
O_{(2 n+1) m \times m} & I_{(2 n+1) m} \\
O_{m \times m} & O_{m \times(2 n+1) m}
\end{array}\right), \quad v=\left(\begin{array}{c}
I_{m} \\
O_{(2 n+1) m \times m}
\end{array}\right), \\
& u=\operatorname{col}\left(O-s_{0} O-s_{1} \ldots-s_{n}\right) \in \mathbb{C}^{m \times(2 n+2) m}, \quad R(w)=\left(I_{(2 n+2) m}-w T\right)^{-1}, \\
& H_{1}=\left(s_{j+k}\right)_{j, k=0}^{n}, \quad H_{2}=\left(s_{j+k+1}\right)_{j, k=0}^{n} \text {, } \\
& T_{1}=\left(\begin{array}{cc}
O_{m n \times m} & I_{m n} \\
O_{m \times m} & O_{m \times m n}
\end{array}\right), \quad v_{1}=\left(\begin{array}{c}
I_{m} \\
O_{m n \times m}
\end{array}\right) \text {, } \\
& R_{1}(w)=\left(I_{(n+1) m}-w T_{1}\right)^{-1}, \quad u_{2}=\operatorname{col}\left(-s_{0}-s_{1} \ldots-s_{n}\right), \quad u_{1}=T_{1}^{*} u_{2} .
\end{aligned}
$$

It is easily verified that the block matrices defined above satisfy the main identity

$$
T_{1}^{*} H_{2}-H_{1}=v_{1} u_{2}^{*}
$$

By $P$ denote block matrix

$$
P=\left(\begin{array}{ccccccc}
I & O & O & O & \ldots & O & O \\
O & O & I & O & \ldots & O & O \\
\vdots & \vdots & \vdots & \vdots & \ddots & \vdots & \vdots \\
O & O & O & O & \ldots & I & O \\
O & I & O & O & \ldots & O & O \\
O & O & O & I & \ldots & O & O \\
\vdots & \vdots & \vdots & \vdots & \ddots & \vdots & \vdots \\
O & O & O & O & \ldots & O & I
\end{array}\right) \in \mathbb{C}^{(2 n+2) m \times(2 n+2) m}
$$

These matrix has exactly one entry of 1 in each row and each column and 0 s elsewhere, i.e., $P$ is a permutation matrix. In particular, the matrix $P$ is orthogonal $P^{\prime} P=P P^{\prime}=I$. 
It is easy to see that

(15) $P v=\left(\begin{array}{c}v_{1} \\ O_{(n+1) m \times m}\end{array}\right), \quad P u=\left(\begin{array}{c}O_{(n+1) m \times m} \\ u_{2}\end{array}\right)$,

(16) $P H P^{\prime}=\left(\begin{array}{cc}H_{1} & O_{(n+1) m \times(n+1) m} \\ O_{(n+1) m \times(n+1) m} & H_{2}\end{array}\right)$,

(17) $v^{*} R(w) P^{\prime}=\left(\begin{array}{lll}v_{1}^{*} R_{1}\left(w^{2}\right) & w v_{1}^{*} R_{1}\left(w^{2}\right)\end{array}\right), \quad u^{*} R(w) P^{\prime}=\left(\begin{array}{cc}A(w) & u_{2}^{*} R_{1}\left(w^{2}\right)\end{array}\right)$.

Hear by $A(w)$ denote some polynomial $m \times m(n+1) \mathrm{MF}$.

It follows from (13), (16) that

$$
H>O \Leftrightarrow H_{1}>O \wedge H_{2}>O \text {. }
$$

This implies that

$$
H^{-1}=P^{\prime}\left(\begin{array}{cc}
H_{1}^{-1} & O_{(n+1) m \times(n+1) m} \\
O_{(n+1) m \times(n+1) m} & H_{2}^{-1}
\end{array}\right) P .
$$

Theorem 3. Let

$$
U(w)=\left(\begin{array}{cc}
\alpha(w) & \beta(w) \\
\gamma(w) & \delta(w)
\end{array}\right)=\left(\begin{array}{cc}
I+w v^{*} R(w) H^{-1} u & -w v^{*} R(w) H^{-1} v \\
w u^{*} R(w) H^{-1} u & I-w u^{*} R(w) H^{-1} v
\end{array}\right)
$$

be a resolvent matrix for the Hamburger symmetric matrix moment problem that corresponds to sequence (12). Then

$$
\begin{aligned}
U(w) & =\left(\begin{array}{cc}
I+w^{2} v_{1}^{*} R_{1}\left(w^{2}\right) H_{2}^{-1} u_{2} & -w v_{1}^{*} R_{1}\left(w^{2}\right) H_{1}^{-1} v_{1} \\
w u_{2}^{*} R_{1}\left(w^{2}\right) H_{2}^{-1} u_{2} & I-w^{2} u_{1}^{*} R_{1}\left(w^{2}\right) H_{1}^{-1} v_{1}
\end{array}\right), \\
2 i\left(\bar{w}_{0}\right. & \left.-w_{0}\right) v^{*} R\left(w_{0}\right) H^{-1} R^{*}\left(w_{0}\right) v=v_{1}^{*} R_{1}\left(w_{0}^{2}\right) H_{1}^{-1} R_{1}^{*}\left(w_{0}^{2}\right) v_{1} \\
& +w_{0} \bar{w}_{0} v_{1}^{*} R_{1}\left(w_{0}^{2}\right) H_{2}^{-1} R_{1}^{*}\left(w_{0}^{2}\right) v_{1} .
\end{aligned}
$$

Proof. Using (15), (16), and (17), we get

$$
\begin{aligned}
\alpha(w) & =I+w v^{*} R(w) P^{\prime}\left(\begin{array}{cc}
H_{1}^{-1} & O_{(n+1) m \times(n+1) m} \\
O_{(n+1) m \times(n+1) m} & H_{2}^{-1}
\end{array}\right) P u \\
& =I+w\left(v_{1}^{*} R_{1}\left(w^{2}\right) \quad w v_{1}^{*} R_{1}\left(w^{2}\right)\right)\left(\begin{array}{cc}
H_{1}^{-1} & O \\
O & H_{2}^{-1}
\end{array}\right)\left(\begin{array}{c}
O \\
u_{2}
\end{array}\right) \\
& =I+w^{2} v_{1}^{*} R_{1}\left(w^{2}\right) H_{2}^{-1} u_{2} .
\end{aligned}
$$

Formulas

$$
\begin{aligned}
& \beta(w)=-w v_{1}^{*} R_{1}\left(w^{2}\right) H_{1}^{-1} v_{1}, \\
& \gamma(w)=w u_{2}^{*} R_{1}\left(w^{2}\right) H_{2}^{-1} u_{2}, \\
& \delta(w)=I-w^{2} u_{1}^{*} R_{1}\left(w^{2}\right) H_{1}^{-1} v_{1}
\end{aligned}
$$

and (19) are proved by analogy. This completes the proof of theorem 3.

Lemma 1. Let $U(z)$ be a resolvent matrix for the Hamburger symmetric matrix moment problem that corresponds to sequence (12). Then

$$
U(i y) J_{\pi} U^{*}(i y)-J_{\pi}=O_{2 m \times 2 m}, \quad U^{*}(i y) J_{\pi} U(i y)-J_{\pi}=O_{2 m \times 2 m} \quad \forall y>0 .
$$

Proof. We have

$$
\begin{aligned}
& U(i y) J_{\pi} U^{*}(i y)-J_{\pi}=\left(\begin{array}{cc}
-i y v_{1}^{*} R_{1}\left(-y^{2}\right) H_{1}^{-1} v_{1} & I-y^{2} v_{1}^{*} R_{1}\left(-y^{2}\right) H_{2}^{-1} u_{2} \\
I+y^{2} u_{1}^{*} R_{1}\left(-y^{2}\right) H_{1}^{-1} v_{1} & i y u_{2}^{*} R_{1}\left(-y^{2}\right) H_{2}^{-1} u_{2}
\end{array}\right) \\
& \times\left(\begin{array}{cc}
I-y^{2} u_{2}^{*} H_{2}^{-1} R_{1}^{*}\left(-y^{2}\right) v_{1} & -i y u_{2}^{*} H_{2}^{-1} R_{1}^{*}\left(-y^{2}\right) u_{2} \\
i y v_{1}^{*} H_{1}^{-1} R_{1}^{*}\left(-y^{2}\right) v_{1} & I+y^{2} v_{1}^{*} H_{1}^{-1} R_{1}^{*}\left(-y^{2}\right) u_{1}
\end{array}\right)-J_{\pi}=\left(\begin{array}{cc}
A_{11}(y) & A_{12}(y) \\
A_{21}(y) & A_{22}(y)
\end{array}\right) .
\end{aligned}
$$


Using (14), we get

$$
\begin{aligned}
& A_{11}(y)=\left(-i y v_{1}^{*} R_{1}\left(-y^{2}\right) H_{1}^{-1} v_{1}\right)\left(I-y^{2} u_{2}^{*} H_{2}^{-1} R_{1}^{*}\left(-y^{2}\right) v_{1}\right) \\
& +\left(I-y^{2} v_{1}^{*} R_{1}\left(-y^{2}\right) H_{2}^{-1} u_{2}\right)\left(i y v_{1}^{*} H_{1}^{-1} R_{1}^{*}\left(-y^{2}\right) v_{1}\right) \\
& =-i y v_{1}^{*} R_{1}\left(-y^{2}\right) H_{1}^{-1} v_{1}+i y^{3} v_{1}^{*} R_{1}\left(-y^{2}\right) H_{1}^{-1} v_{1} u_{2}^{*} H_{2}^{-1} R_{1}^{*}\left(-y^{2}\right) v_{1} \\
& +i y v_{1}^{*} H_{1}^{-1} R_{1}^{*}\left(-y^{2}\right) v_{1}-i y^{3} v_{1}^{*} R_{1}\left(-y^{2}\right) H_{2}^{-1} u_{2} v_{1}^{*} H_{1}^{-1} R_{1}^{*}\left(-y^{2}\right) v_{1} \\
& =-i y v_{1}^{*} R_{1}\left(-y^{2}\right) H_{1}^{-1} v_{1}+i y^{3} v_{1}^{*} R_{1}\left(-y^{2}\right) H_{1}^{-1}\left(T_{1}^{*} H_{2}-H_{1}\right) H_{2}^{-1} R_{1}^{*}\left(-y^{2}\right) v_{1} \\
& +i y v_{1}^{*} H_{1}^{-1} R_{1}^{*}\left(-y^{2}\right) v_{1}-i y^{3} v_{1}^{*} R_{1}\left(-y^{2}\right) H_{2}^{-1}\left(H_{2} T_{1}-H_{1}\right) H_{1}^{-1} R_{1}^{*}\left(-y^{2}\right) v_{1} \\
& =i y v_{1}^{*} R_{1}\left(-y^{2}\right) H_{1}^{-1}\left(-R_{1}^{-*}\left(-y^{2}\right)+y^{2}\left(T_{1}^{*} H_{2}-H_{1}\right) H_{2}^{-1}\right) R_{1}^{*}\left(-y^{2}\right) v_{1} \\
& +i y v_{1}^{*} R_{1}\left(-y^{2}\right)\left(R_{1}^{-1}\left(-y^{2}\right)-y^{2} H_{2}^{-1}\left(H_{2} T_{1}-H_{1}\right)\right) H_{1}^{-1} R_{1}^{*}\left(-y^{2}\right) v_{1} \\
& =i y v_{1}^{*} R_{1}\left(-y^{2}\right) H_{1}^{-1}\left(-I-y^{2} T_{1}^{*}+y^{2} T_{1}^{*}-y^{2} H_{1} H_{2}^{-1}\right) R_{1}^{*}\left(-y^{2}\right) v_{1} \\
& +i y v_{1}^{*} R_{1}\left(-y^{2}\right)\left(I+y^{2} T_{1}-y^{2} T_{1}+y^{2} H_{2}^{-1} H_{1}\right) H_{1}^{-1} R_{1}^{*}\left(-y^{2}\right) v_{1} \\
& =-i y v_{1}^{*} R_{1}\left(-y^{2}\right)\left(H_{1}^{-1}+y^{2} H_{2}^{-1}\right) R_{1}^{*}\left(-y^{2}\right) v_{1} \\
& +i y v_{1}^{*} R_{1}\left(-y^{2}\right)\left(H_{1}^{-1}+y^{2} H_{2}^{-1}\right) R_{1}^{*}\left(-y^{2}\right) v_{1}=O_{m \times m} .
\end{aligned}
$$

Thus we have $A_{11}(y)=O_{m \times m}$. The equalities $A_{12}(y)=A_{21}(y)=A_{22}(y)=O_{m \times m}$ are proved in a similar way. Finally, we obtain $U(i y) J_{\pi} U^{*}(i y)-J_{\pi}=O_{2 m \times 2 m}$. It follows (see, for example, [1]) that $U^{*}(i y) J_{\pi} U(i y)-J_{\pi}=O_{2 m \times 2 m}$. Lemma 1 is proved.

A Nevanlinna pair col $(p(w) q(w))$ is called symmetric if

$$
\left(p^{*}(i y) q^{*}(i y)\right) J_{\pi}\left(\begin{array}{c}
p(i y) \\
q(i y)
\end{array}\right)=O, \quad y>0 .
$$

If some Nevanlinna pair is symmetric then all equivalent Nevanlinna pairs is also symmetric. The corresponding equivalence class of Nevanlinna pairs is called symmetric. The set of equivalence classes of Nevanlinna symmetric pairs will be denoted by $\tilde{\mathcal{R}}_{\infty}$.

The Nevanlinna MF $f(w) \in \mathcal{R}$ is called symmetric if Nevanlinna pair col $(I f(w))$ is symmetric, i.e.,

$$
f(i y)+f^{*}(i y)=O, \quad y>0 .
$$

The set of Nevanlinna symmetric MF will be denoted by $\tilde{\mathcal{R}}$.

Consider the symmetric Hamburger moment problem. By definition, put

$$
\tilde{\mathcal{F}}=\{f(w) \in \mathcal{F} \mid f(w) \in \tilde{\mathcal{R}}\}
$$

Theorem 4. Let

$$
U(w)=\left(\begin{array}{cc}
\alpha(w) & \beta(w) \\
\gamma(w) & \delta(w)
\end{array}\right)=\left(\begin{array}{cc}
I+w^{2} v_{1}^{*} R_{1}\left(w^{2}\right) H_{2}^{-1} u_{2} & -w v_{1}^{*} R_{1}\left(w^{2}\right) H_{1}^{-1} v_{1} \\
w u_{2}^{*} R_{1}\left(w^{2}\right) H_{2}^{-1} u_{2} & I-w^{2} u_{1}^{*} R_{1}\left(w^{2}\right) H_{1}^{-1} v_{1}
\end{array}\right)
$$

be a resolvent matrix for the Hamburger symmetric matrix moment problem that corresponds to sequence (12). Then the formula

$$
f(w)=(\gamma(w) p(w)+\delta(w) q(w))(\alpha(w) p(w)+\beta(w) q(w))^{-1}
$$

establishes a bijective correspondence between $\tilde{\mathcal{F}}$ and $\tilde{\mathcal{R}}_{\infty}$. 
Proof. By $f, p, q, \alpha, \beta, \gamma, \delta$ denote $f(i y), p(i y), q(i y), \alpha(i y), \beta(i y), \gamma(i y), \delta(i y), \quad y>0$ respectively. We have

$$
\begin{aligned}
& f+f^{*}=\left(\begin{array}{ll}
I & f^{*}
\end{array}\right) J_{\pi}\left(\begin{array}{c}
I \\
f
\end{array}\right) \\
& =\left(\begin{array}{ll}
I & (\alpha p+\beta q)^{-*}\left(p^{*} \gamma^{*}+q^{*} \delta^{*}\right)
\end{array}\right) J_{\pi}\left(\begin{array}{c}
I \\
(\gamma p+\delta q)(\alpha p+\beta q)^{-1}
\end{array}\right)
\end{aligned}
$$

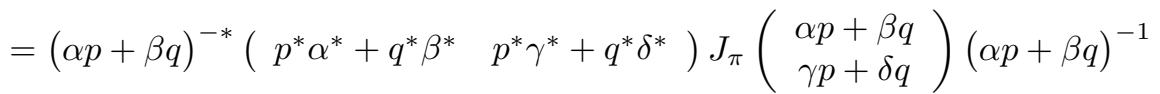

$$
\begin{aligned}
& =(\alpha p+\beta q)^{-*}\left(\begin{array}{cc}
p^{*} & q^{*}
\end{array}\right) U^{*} J_{\pi} U\left(\begin{array}{c}
p \\
q
\end{array}\right)(\alpha p+\beta q)^{-1} \\
& =(\alpha p+\beta q)^{-*}\left(\begin{array}{cc}
p^{*} & q^{*}
\end{array}\right) J_{\pi}\left(\begin{array}{c}
p \\
q
\end{array}\right)(\alpha p+\beta q)^{-1} \text {. }
\end{aligned}
$$

Thus

$$
\begin{aligned}
& f(i y)+f^{*}(i y)=(\alpha(i y) p(i y)+\beta(i y) q(i y))^{-*} \\
& \times\left(\begin{array}{ll}
p^{*}(i y) & \left.q^{*}(i y)\right) J_{\pi}\left(\begin{array}{c}
p(i y) \\
q(i y)
\end{array}\right)(\alpha(i y) p(i y)+\beta(i y) q(i y))^{-1}, \quad y>0 .
\end{array}\right.
\end{aligned}
$$

This implies that

$$
f \in \tilde{\mathcal{F}} \Leftrightarrow \operatorname{col}\left(\begin{array}{ll}
p & g
\end{array}\right) Q \in \tilde{\mathcal{R}}_{\infty} .
$$

Theorem 4 is proved.

Denote by $\tilde{\mathcal{I}}(f ; i y), y>0$ the restriction of the entropy functional $(10)$ to $\tilde{\mathcal{F}} \subset \mathcal{F}$.

Theorem 5. The entropy functional has an upper bound

$$
\begin{aligned}
\tilde{\mathcal{I}}(f ; i y) & \leq \ln \operatorname{det}\left(4 y \left(v_{1}^{*} R_{1}\left(-y^{2}\right) H_{1}^{-1} R_{1}^{*}\left(-y^{2}\right) v_{1}\right.\right. \\
& \left.\left.+y^{2} v_{1}^{*} R_{1}\left(-y^{2}\right) H_{2}^{-1} R_{1}^{*}\left(-y^{2}\right) v_{1}\right)\right)^{-1}
\end{aligned}
$$

for all $f \in \tilde{\mathcal{F}}$ with equality if and only if

$$
\begin{gathered}
\tilde{f}(w)=(\gamma(w) \tilde{p}(w)+\delta(w) \tilde{q}(w))(\alpha(w) \tilde{p}(w)+\beta(w) \tilde{q}(w))^{-1} \in \tilde{\mathcal{F}}, \\
\left(\begin{array}{c}
\tilde{p}(w) \\
\tilde{q}(w)
\end{array}\right)=\left(\begin{array}{c}
-\beta^{*}(i y) \\
\alpha^{*}(i y)
\end{array}\right) Q(w) \in \tilde{\mathcal{R}}_{\infty} .
\end{gathered}
$$

Proof. Using (11), (19), we get (22). Next we have

$$
\begin{aligned}
\left(\begin{array}{rl}
\tilde{p}^{*}(w) & \tilde{q}^{*}(w)
\end{array}\right) J_{\pi}\left(\begin{array}{c}
\tilde{p}(w) \\
\tilde{q}(w)
\end{array}\right) & =Q^{*}(w)\left(\begin{array}{ll}
-\beta(i y) & \alpha(i y)
\end{array}\right) J_{\pi}\left(\begin{array}{c}
-\beta^{*}(i y) \\
\alpha^{*}(i y)
\end{array}\right) Q(w) \\
& =Q^{*}(w)\left(-\beta(i y) \alpha^{*}(i y)-\alpha(i y) \beta^{*}(i y)\right) Q(w)=O .
\end{aligned}
$$

Here we used the formula (20). Thus

$$
\left(\begin{array}{c}
-\beta^{*}(i y) \\
\alpha^{*}(i y)
\end{array}\right) Q(w) \in \tilde{\mathcal{R}}_{\infty} .
$$

Using (23), Theorem 4, and Theorem 2, we get Theorem 5 . 


\section{Entropy functionals for the Stieltjes moment Problem}

Let

$$
s_{0}, O, s_{1}, O, s_{2}, O, \ldots, O, s_{2 n+1} \text {. }
$$

be a sequence of $m \times m$ matrices such that block matrix $H$ (see (13)) is positive. Consider the Hamburger symmetric matrix moment problem that corresponds to sequence (24) and denote by $\tilde{\mathcal{F}}$ the set of symmetric associate MF.

Consider the sequence

$$
s_{0}, s_{1}, \ldots, s_{2 n+1} \text {. }
$$

Using (18), we get $H_{1}>O, H_{2}>O$. Denote by $\mathcal{F}_{+}$the set MF that are associated with the Stieltjes matrix moment problem

$$
s_{j}=\int_{\mathbb{R}_{+}} t^{j} \sigma(d t), \quad 0 \leq j \leq 2 n, \quad s_{2 n+1} \geq \int_{\mathbb{R}_{+}} t^{2 n+1} \sigma(d t) .
$$

Lemma 2. The formula

$$
\left.\left(\begin{array}{c}
p_{1}(w) \\
\frac{1}{w} g_{1}(w)
\end{array}\right)\right|_{w^{2}=z}=\left(\begin{array}{c}
p(z) \\
g(z)
\end{array}\right)
$$

establishes a bijective correspondence between $\tilde{\mathcal{R}}_{\infty}$ and $\mathcal{S}_{\infty}$.

Moreover, the formula

$$
\left.\frac{1}{w} f(w)\right|_{w^{2}=z}=s(z)
$$

establishes a bijective correspondence between $\tilde{\mathcal{F}}$ and $\mathcal{F}_{+}$.

Proof. The first statement of the lemma is obvious (see [12], [14]). Let us prove the second statement of the lemma. If $f \in \tilde{\mathcal{F}}$, then there exists a pair $\operatorname{col}\left(p_{1}(w) g_{1}(w)\right) \in \tilde{\mathcal{R}}_{\infty}$ such that $f(w)=\left(\gamma(w) p_{1}(w)+\delta(w) q_{1}(w)\right)\left(\alpha(w) p_{1}(w)+\beta(w) q_{1}(w)\right)^{-1}$.

Consequently

$$
\begin{aligned}
& \left.\frac{1}{w} f(w)\right|_{w^{2}=z}=\left.\frac{1}{w}\left(\gamma(w) p_{1}(w)+\delta(w) q_{1}(w)\right)\left(\alpha(w) p_{1}(w)+\beta(w) q_{1}(w)\right)^{-1}\right|_{w^{2}=z} \\
& =\left.\left(\frac{\gamma(w)}{w} p_{1}(w)+\delta(w) \frac{q_{1}(w)}{w}\right)\left(\alpha(w) p_{1}(w)+w \beta(w) \frac{q_{1}(w)}{w}\right)^{-1}\right|_{w^{2}=z} \\
& =\left(\frac{w u_{2}^{*} R_{1}\left(w^{2}\right) H_{2}^{-1} u_{2}}{w} p_{1}(w)+\left(I-w^{2} u_{1}^{*} R_{1}\left(w^{2}\right) H_{1}^{-1} v_{1}\right) \frac{q_{1}(w)}{w}\right) \\
& \times\left.\left(\left(I+w^{2} v_{1}^{*} R_{1}\left(w^{2}\right) H_{2}^{-1} u_{2}\right) p_{1}(w)+\left(-w^{2} v_{1}^{*} R_{1}\left(w^{2}\right) H_{1}^{-1} v_{1}\right) \frac{q_{1}(w)}{w}\right)^{-1}\right|_{w^{2}=z} \\
& =\left(\gamma_{1}(z) p(z)+\delta_{1}(z) q(z)\right)\left(\alpha_{1}(z) p(z)+\beta_{1}(z) q(z)\right)^{-1}=s(z) \in \mathcal{F}_{+} .
\end{aligned}
$$

Lemma 2 is proved.

Proof of Theorem 1. The proof is divided into steps.

Step 1. If $f(w)$ and $s(z)$ are as in Lemma 2 and

$$
\tilde{\mathcal{I}}\left(f ; i y_{0}\right)=\int_{\mathbb{R}} \ln \left(\operatorname{det} \frac{f(x)-f^{*}(x)}{2 i}\right) \frac{y_{0}}{\pi\left(x^{2}+y_{0}^{2}\right)} d x, \quad y_{0}>0,
$$

then

(25) $\quad \tilde{\mathcal{I}}\left(f ; i y_{0}\right)=\frac{m}{2} \ln \left(-t_{0}\right)+\int_{0}^{\infty} \ln \operatorname{det}\left\{\frac{s(t)-s^{*}(t)}{2 i}\right\} \cdot \frac{\sqrt{-t_{0}}}{\pi\left(t-t_{0}\right)} \frac{d t}{\sqrt{t}}, \quad t_{0}=-y_{0}^{2}$. 
Proof of step 1. We have

$$
\begin{aligned}
\tilde{\mathcal{I}}\left(f ; i y_{0}\right) & =\int_{\mathbb{R}} \ln \left(\operatorname{det} \frac{f(x)-f^{*}(x)}{2 i}\right) \frac{y_{0}}{\pi\left(x^{2}+y_{0}^{2}\right)} d x \\
& =2 \int_{\mathbb{R}_{+}} \ln \left(\operatorname{det} x \frac{\frac{f(x)}{x}-\frac{f^{*}(x)}{x}}{2 i}\right) \frac{y_{0}}{\pi\left(x^{2}+y_{0}^{2}\right)} d x .
\end{aligned}
$$

Substituting $x^{2}$ for $t$ in the last integral, we get

$$
\tilde{\mathcal{I}}\left(f ; i y_{0}\right)=\frac{m}{2} \int_{0}^{\infty} \ln (t) \cdot \frac{\sqrt{-t_{0}}}{\pi\left(t-t_{0}\right)} \frac{d t}{\sqrt{t}}+\int_{0}^{\infty} \ln \operatorname{det}\left\{\frac{s(t)-s(t)^{*}}{2 i}\right\} \cdot \frac{\sqrt{-t_{0}}}{\pi\left(t-t_{0}\right)} \frac{d t}{\sqrt{t}} .
$$

But

$$
\frac{m}{2} \int_{0}^{\infty} \ln (t) \cdot \frac{\sqrt{-t_{0}}}{\pi\left(t-t_{0}\right)} \frac{d t}{\sqrt{t}}=\frac{m}{2} \ln \left(-t_{0}\right) .
$$

Indeed, we get this result if substituting $\sqrt{t}$ for $x$ in the last integral and using the formula (see [11], p. 546)

$$
\int_{0}^{\infty} \frac{\ln x d x}{x^{2}+a^{2}}=\frac{\pi}{2 a} \ln a, \quad a>0
$$

Combining last formulas, we get (25).

Step 2. There is an inequality

$$
\int_{0}^{\infty} \ln \operatorname{det}\left\{\frac{s(t)-s(t)^{*}}{2 i}\right\} \cdot \frac{\sqrt{-t_{0}}}{\pi\left(t-t_{0}\right)} \frac{d t}{\sqrt{t}} \leq \ln \operatorname{det} \frac{s_{K}\left(t_{0}\right)-s_{F}\left(t_{0}\right)}{4} \quad \forall s \in \mathcal{F}_{+} .
$$

Proof of step 2. Using Theorem 5 and (25), we get

$$
\begin{aligned}
& \frac{m}{2} \ln \left(-t_{0}\right)+\int_{0}^{\infty} \ln \operatorname{det}\left\{\frac{s(t)-s^{*}(t)}{2 i}\right\} \cdot \frac{\sqrt{-t_{0}}}{\pi\left(t-t_{0}\right)} \frac{d t}{\sqrt{t}} \\
& \quad \leq \ln \operatorname{det}\left(4 \sqrt{-t_{0}}\left(v_{1}^{*} R_{1}\left(t_{0}\right) H_{1}^{-1} R_{1}^{*}\left(t_{0}\right) v_{1}-t_{0} v_{1}^{*} R_{1}\left(t_{0}\right) H_{2}^{-1} R_{1}^{*}\left(t_{0}\right) v_{1}\right)\right)^{-1} .
\end{aligned}
$$

From [6], we get the following formula:

$$
\left(v_{1}^{*} R_{1}\left(t_{0}\right) H_{1}^{-1} R_{1}^{*}\left(t_{0}\right) v_{1}-t_{0} \bar{w}_{0} v_{1}^{*} R_{1}\left(t_{0}\right) H_{2}^{-1} R_{1}^{*}\left(t_{0}\right) v_{1}\right)^{-1}=-t_{0}\left(s_{K}\left(t_{0}\right)-s_{F}\left(t_{0}\right)\right) .
$$

This yields that

$$
\begin{aligned}
\frac{m}{2} \ln ( & \left.-t_{0}\right)+\int_{0}^{\infty} \ln \operatorname{det}\left\{\frac{s(t)-s^{*}(t)}{2 i}\right\} \cdot \frac{\sqrt{-t_{0}}}{\pi\left(t-t_{0}\right)} \frac{d t}{\sqrt{t}} \\
& \leq \ln \operatorname{det}\left(\left(4 \sqrt{-t_{0}}\right)^{-1}\left(-t_{0}\right)\left(s_{K}\left(t_{0}\right)-s_{F}\left(t_{0}\right)\right)=\ln \operatorname{det}\left(\sqrt{-t_{0}} \frac{s_{K}\left(t_{0}\right)-s_{F}\left(t_{0}\right)}{4}\right)\right. \\
& =\ln \left(\left(-t_{0}\right)^{\frac{m}{2}} \operatorname{det} \frac{s_{K}\left(t_{0}\right)-s_{F}\left(t_{0}\right)}{4}\right)=\frac{m}{2} \ln \left(-t_{0}\right)+\ln \operatorname{det} \frac{s_{K}\left(t_{0}\right)-s_{F}\left(t_{0}\right)}{4} .
\end{aligned}
$$

It immediately follows (26).

Step 3. In inequality (26) we have equality if and only if

$$
\begin{gathered}
\tilde{s}(z)=\left(\gamma_{1}(z) \tilde{p}(z)+\delta_{1}(z) \tilde{q}(z)\right)\left(\alpha_{1}(z) \tilde{p}(z)+\beta_{1}(z) \tilde{q}(z)\right)^{-1} \\
\left(\begin{array}{c}
\tilde{p}(z) \\
\tilde{q}(z)
\end{array}\right)=\left(\begin{array}{c}
\beta_{1}^{*}\left(t_{0}\right) / \sqrt{t_{0}} \\
\alpha_{1}^{*}\left(t_{0}\right) / \sqrt{z}
\end{array}\right) Q(z) .
\end{gathered}
$$

Proof of step 3. Using (5) and (21), we get

$$
\begin{gathered}
\alpha\left(i y_{0}\right)=I+\left(-y_{0}^{2}\right) v_{1}^{*} R_{1}\left(-y_{0}^{2}\right) H_{2}^{-1} u_{2}=I+t_{0} v_{1}^{*} R_{1}\left(t_{0}\right) H_{2}^{-1} u_{2}=\alpha_{1}\left(t_{0}\right), \\
-\beta\left(i y_{0}\right)=-i y_{0} v_{1}^{*} R_{1}\left(-y_{0}^{2}\right) H_{1}^{-1} v_{1}=-\sqrt{t_{0}} v_{1}^{*} R_{1}\left(t_{0}\right) H_{1}^{-1} v_{1}=\frac{\beta_{1}\left(t_{0}\right)}{\sqrt{t_{0}}} .
\end{gathered}
$$


Using Theorem 5, we get

$$
\begin{aligned}
\tilde{s}(z) & =\left.\frac{1}{w} \tilde{f}(w)\right|_{w^{2}=z} \\
& =\left.\frac{1}{w}\left(\gamma(w)\left(-\beta^{*}\left(i y_{0}\right)\right)+\delta(w) \alpha^{*}\left(i y_{0}\right)\right)\left(\alpha(w)\left(-\beta^{*}\left(i y_{0}\right)\right)+\beta(w) \alpha^{*}\left(i y_{0}\right)\right)^{-1}\right|_{w^{2}=z} \\
& =\left.\left(\frac{\gamma(w)}{w}\left(-\beta^{*}\left(i y_{0}\right)\right)+\delta(w) \frac{\alpha^{*}\left(i y_{0}\right)}{w}\right)\left(\alpha(w)\left(-\beta^{*}\left(i y_{0}\right)\right)+w \beta(w) \frac{\alpha^{*}\left(i y_{0}\right)}{w}\right)^{-1}\right|_{w^{2}=z} \\
& =\left(\frac{w u_{2}^{*} R_{1}\left(w^{2}\right) H_{2}^{-1} u_{2}}{w}\left(-\beta^{*}\left(i y_{0}\right)\right)+\left(I-w^{2} u_{1}^{*} R_{1}\left(w^{2}\right) H_{1}^{-1} v_{1}\right) \frac{\alpha^{*}\left(i y_{0}\right)}{w}\right) \\
& \times\left.\left(\left(I+w^{2} v_{1}^{*} R_{1}\left(w^{2}\right) H_{2}^{-1} u_{2}\right)\left(-\beta^{*}\left(i y_{0}\right)\right)+\left(-w^{2} v_{1}^{*} R_{1}\left(w^{2}\right) H_{1}^{-1} v_{1}\right) \frac{\alpha^{*}\left(i y_{0}\right)}{w}\right)^{-1}\right|_{w^{2}=z} \\
& =\left(\gamma_{1}(z) \frac{\beta_{1}^{*}\left(t_{0}\right)}{\sqrt{t_{0}}}+\delta_{1}(z) \frac{\alpha_{1}^{*}\left(t_{0}\right)}{\sqrt{z}}\right)\left(\alpha_{1}(z) \frac{\beta_{1}^{*}\left(t_{0}\right)}{\sqrt{t_{0}}}+\beta_{1}(z) \frac{\alpha_{1}^{*}\left(t_{0}\right)}{\sqrt{z}}\right)^{-1} \cdot
\end{aligned}
$$

Thus

$$
\begin{gathered}
\tilde{s}(z)=\left(\gamma_{1}(z) \tilde{p}(z)+\delta_{1}(z) \tilde{q}(z)\right)\left(\alpha_{1}(z) \tilde{p}(z)+\beta_{1}(z) \tilde{q}(z)\right)^{-1} \\
\left(\begin{array}{c}
\tilde{p}(z) \\
\tilde{q}(z)
\end{array}\right)=\left(\begin{array}{c}
\beta_{1}^{*}\left(t_{0}\right) / \sqrt{t_{0}} \\
\alpha_{1}^{*}\left(t_{0}\right) / \sqrt{z}
\end{array}\right) Q(z) .
\end{gathered}
$$

Step 4 completes the proof.

By [6], it follows that

$$
\begin{gathered}
\left(\begin{array}{ll}
\alpha_{1}\left(t_{0}\right) & \beta_{1}\left(t_{0}\right) \\
\gamma_{1}\left(t_{0}\right) & \delta_{1}\left(t_{0}\right)
\end{array}\right)\left(\begin{array}{cc}
O & I \\
I & O
\end{array}\right)\left(\begin{array}{cc}
\alpha_{1}\left(t_{0}\right) & \beta_{1}\left(t_{0}\right) \\
\gamma_{1}\left(t_{0}\right) & \delta_{1}\left(t_{0}\right)
\end{array}\right)^{*}-\left(\begin{array}{cc}
O & I \\
I & O
\end{array}\right) \\
=2\left(\begin{array}{cc}
\left(s_{K}\left(t_{0}\right)-s_{F}\left(t_{0}\right)\right)^{-1} & \left(s_{K}\left(t_{0}\right)-s_{F}\left(t_{0}\right)\right)^{-1} s_{F}\left(t_{0}\right) \\
s_{F}\left(t_{0}\right)\left(s_{K}\left(t_{0}\right)-s_{F}\left(t_{0}\right)\right)^{-1} & \left(s_{F}^{-1}\left(t_{0}\right)-s_{K}^{-1}\left(t_{0}\right)\right)^{-1}
\end{array}\right)
\end{gathered}
$$

Hence

$$
\begin{aligned}
& \alpha_{1}\left(t_{0}\right) \beta_{1}^{*}\left(t_{0}\right)+\beta_{1}\left(t_{0}\right) \alpha_{1}^{*}\left(t_{0}\right)=2\left(s_{K}\left(t_{0}\right)-s_{F}\left(t_{0}\right)\right)^{-1}, \\
& \gamma_{1}\left(t_{0}\right) \beta_{1}^{*}\left(t_{0}\right)+\delta_{1}\left(t_{0}\right) \alpha_{1}^{*}\left(t_{0}\right)=2 s_{F}\left(t_{0}\right)\left(s_{K}\left(t_{0}\right)-s_{F}\left(t_{0}\right)\right)^{-1}+I \\
& \quad=\left(2 s_{F}\left(t_{0}\right)+\left(s_{K}\left(t_{0}\right)-s_{F}\left(t_{0}\right)\right)\right)\left(s_{K}\left(t_{0}\right)-s_{F}\left(t_{0}\right)\right)^{-1} \\
& \quad=\left(s_{K}\left(t_{0}\right)+s_{F}\left(t_{0}\right)\right)\left(s_{K}\left(t_{0}\right)-s_{F}\left(t_{0}\right)\right)^{-1} .
\end{aligned}
$$

Finally, we obtain

$$
\begin{aligned}
\tilde{s}\left(t_{0}\right) & =\left(\gamma_{1}\left(t_{0}\right) \beta_{1}^{*}\left(t_{0}\right)+\delta_{1}\left(t_{0}\right) \alpha_{1}^{*}\left(t_{0}\right)\right)\left(\alpha_{1}\left(t_{0}\right) \beta_{1}^{*}\left(t_{0}\right)+\beta_{1}\left(t_{0}\right) \alpha_{1}^{*}\left(t_{0}\right)\right)^{-1} \\
& =\left(s_{K}\left(t_{0}\right)+s_{F}\left(t_{0}\right)\right)\left(s_{K}\left(t_{0}\right)-s_{F}\left(t_{0}\right)\right)^{-1}\left(s_{K}\left(t_{0}\right)-s_{F}\left(t_{0}\right)\right) / 2=\left(s_{K}\left(t_{0}\right)+s_{F}\left(t_{0}\right)\right) / 2 .
\end{aligned}
$$

Theorem 1 is proved.

\section{REFERENCES}

1. D. Z. Arov, H. Dym, J-Contractive Matrix Valued Functions and Related Topics, Encyclopedia Math. Appl., Cambridge University Press, Cambridge, 2008.

2. D. Z. Arov and M. G. Krein, The problem of finding the minimum entropy in indeterminate problems of continuation, Funktsional. Anal. i Prilozhen. 15 (1981), no. 2, 61-64. (Russian)

3. D. Z. Arov and M. G. Krein, Calculation of entropy functionals and their minima in indeterminate continuation problems, Acta Sci. Math. (Szeged) 45 (1983), no. 1-4, 33-50. (Russian)

4. Ju. M. Berezanskii, Expansions in Eigenfunctions of Selfadjoint Operators, Naukova Dumka, Kiev, 1965. (Russian); English transl. Translations of Mathematical Monographs, 17, American Mathematical Society, Providence, RI, 1968. 
5. H. Dym, On Hermitian block Hankel matrices, matrix polynomials, the Hamburger moment problem, interpolation and maximum entropy, Integr. Equ. Oper. Theory 12 (1989), no. 6, $757-812$.

6. Yu. M. Dyukarev, Indeterminacy criteria for the Stieltjes matrix moment problem, Mat. Zametki 75 (2004), no. 1-2, 71-88. (Russian); English transl. Math. Notes 75 (2004), no. 1-2, 66-82.

7. Yu. M. Dyukarev, Indeterminacy of interpolation problems in the Stieltjes class, Mat. Sb. 196 (2005), no. 3, 61-88. (Russian); English transl. Sb. Math 196 (2005), no. 3, 367-393.

8. Yu. M. Dyukarev, Deficiency numbers of symmetric operators generated by block Jacobi matrices, Mat. Sb. 197 (2006), no. 8, 73-100. (Russian); English transl. Sb. Math 197 (2006), no. 8, 1177-1203.

9. Yu. M. Dyukarev, A generalized Stieltjes criterion for the complete indeterminacy of interpolation problems, Mat. Zametki 84 (2008), no. 1-2, 23-39. (Russian); English transl. Math. Notes 84 (2008), no. 1-2, 22-37.

10. Yu. M. Dyukarev, Geometric and operator measures of degeneracy for the set of solutions to the Stieltjes matrix moment problem, Mat. Sb. 207 (2016), no. 4, 47-64. (Russian); English transl. Sb. Math. 207 (2016), no. 4, 519-536.

11. I. S. Gradshteyn, I. M. Ryzhik, Table of Integrals, Series and Products, Fizmatgiz, Moscow, 1963. (Russian)

12. I. V. Kovalishina, J-expansive matrix-valued functions, and the classical problem of moments, Akad. Nauk Armjan. SSR Dokl. LX (1975), no. 1, 7-11. (Russian)

13. I. V. Kovalishina, Analytic theory of a class of interpolation problems, Izv. Akad. Nauk SSSR Ser. Mat. 47 (1983), no. 3, 455-497. (Russian); English transl. Math. USSR-Izv. 22 (1984), no. 3, 419-463.

14. M. G. Krein, A. A. Nudelman, The Markov Moment Problem and Extremal Problems, Nauka, Moscow, 1973. (Russian); English transl. Translations of Mathematical Monographs, 50, American Mathematical Society, Providence, RI, 1977.

Department of Physics, V. N. Karazin Kharkov National University, Svobody Square 4 , KHARKOV, 61202, UKRAINE

E-mail address: yu.dyukarev@karazin.ua

Received 12/10/2019; Revised 25/10/2019 